\title{
A DISCRETE-TIME DECOUPLING SCHEME FOR A DIFFERENTIALLY CROSS-COUPLED SYSTEM
}

\author{
B. Srinivasan, P. Mullhaupt, T. Baumann and D. Bonvin \\ Institut d'Automatique, Ecole Polytechnique Fédérale de Lausanne, \\ CH-1015 Lausanne, Switzerland. \\ e-mail : name@ia.epfl.ch
}

\begin{abstract}
Problems with differential cross-coupling can arise in mechanical systems when independent external forces are absent in some of the coordinates. Input-output linearization of these systems, in general, leads to unstable internal dynamics. A discrete-time decoupling scheme which circumvents this problem is developed. It is shown that internally-stable decoupling is possible with the proposed method if and only if the sampling interval is larger than a pre-specified value. The design of a stabilizing controller with such a decoupling scheme is also addressed. Simulation results are presented using a helicopter model in which the speed of the propeller is manipulated to control the aerodynamic force.
\end{abstract}

Keywords. Decoupling, Sample-data control, Linearization, Aerospace control

\section{INTRODUCTION}

Control of coupled mechanical systems has been a major area of interest for many a decade. Coupling in these systems is intrinsic and this fact has forced designers to use sophisticated control methodologies. Feedback linearization (Nijmeier and van der Schaft 1990), passivity-based control (Byrnes et al. 1991), integral back-stepping (Kokotovic 1992), and methods exploiting flatness properties (Fliess et al. 1995) are a few among the vast spectrum of techniques used in this context.

In most systems, as in the case of serial robots, it is assumed that an independent external (electric) force can be applied for every coordinate (Ortega and Spong 1989). Also, this applied force is assumed to be large enough to compensate any coupling effects present and drive the system to the desired state. If an independently applied force is absent for one of the axes, the control issue is phenomenally different.

For the purpose of the present development, we will classify the coupling forces acting on an unactuated system coordinate into two categories: (i) the state-dependent forces (e.g. coriolis, centrifugal, and aerodynamic forces) which depend on the speeds and (ii) the accelerationdependent forces (effect of the other applied forces caused by inertial coupling).

The systems considered in this study are those where the state-dependent forces dominate the inertial coupling effects, as is the case in many aerodynamic problems. We will term the coupling of the second type 'differential', due to its relationship with the dominant former. The control design becomes involved, especially when the differential coupling term corresponding to one of the axes appears along with the state-dependent coupling term of another and vice-versa. Such a coupling will be referred to as differential cross-coupling, in the presence of which, input-output linearization using well-known techniques (Isidori et al. 1981) result in unstable internal dynamics. Decoupling is then no longer straightforward, and the design of stabilizing controllers using standard methods becomes extremely cumbersome.

In this work, a discrete-time decoupling method which handles the internal stability problem is proposed. The analysis of this scheme (using a simplified model) leads to a lower bound on the sampling interval. It is also shown that a stabilizing controller can be designed with such a decoupling, if the lower bound mentioned above is smaller than the maximum sampling interval specified by Shannon's sampling theorem (Franklin et al. 1990). The results are then extended to the complete model of a differentially cross-coupled helicopter.

Section 2 is devoted to describing the experimental setup and developing a simplified linear model for the same. The discrete-time decoupling scheme is introduced in Section 3 and the lower bound for the sampling time quantified. Section 4 deals with the effect of various terms present in the complete model. Simulation results which show the importance of the lower bound are depicted in Section 5. The conclusions and open problems are presented in Section 6 . 


\section{A DIFFERENTIALLY CROSS-COUPLED SYSTEM}

Let us first consider the aerodynamic force caused by a rotating propeller. The force depends on (i) the speed of rotation and (ii) the angle of attack of the propeller. In a helicopter, the aerodynamic force is normally controlled by changing the angle of attack, in which case a differential coupling is absent (Prouty 1986). The system is then feedback linearizable, and standard control techniques can be applied (Meyer et al. 1984). In contrast, if the aerodynamic force is controlled by changing the speed of rotation, the reaction of the force applied to change the speed of the propeller causes the body of the helicopter to rotate in an opposite direction. This differential coupling has an extremely adverse effect on the performance.

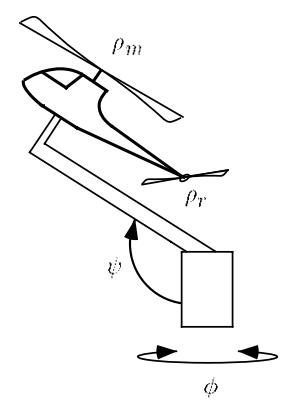

Figure 1. Helicopter Model

The laboratory-scale helicopter model (Figure 1), where the aerodynamic force is controlled by varying the propeller speed, is used for investigation. The setup has two degrees of freedom : the vertical coordinate $\psi$ and the horizontal angle $\phi$. The helicopter system is so constructed that the body of the helicopter can freely rotate in the $\psi$ axis and the stand in the $\phi$ direction. Two electrical drives equipped with propellers perpendicular to each other are mounted on the body. The position of the two blades are $\rho_{m}$ and $\rho_{r}$ and the angles of attack are fixed. It is worthwhile noting that a fixed angle of attack has a decisive advantage in the construction of such small aerodynamic setups.

\subsection{Modeling}

Neglecting friction and other effects, the following simplified model can be written :

$$
\begin{aligned}
I_{\psi} \ddot{\psi}+I_{r} \ddot{\rho}_{r} & =C_{m} \dot{\rho}_{m} \\
I_{\phi} \ddot{\phi}+I_{m} \ddot{\rho}_{m} & =C_{r} \dot{\rho}_{r} \\
I_{m} \ddot{\rho}_{m} & =K_{m} u_{m} \\
I_{r} \ddot{\rho}_{r} & =K_{r} u_{r}
\end{aligned}
$$

The aerodynamic force produced by the main motor acts in the $\psi$ direction and that by the rear motor in the $\phi$ direction. Also, the aerodynamic forces are assumed to be linear with respect to the motor speeds, $\dot{\rho}_{m}$ and $\dot{\rho}_{r}$, the proportionality constants being $C_{m}$ and $C_{r}$ respectively. $I_{\psi}$ and $I_{\phi}$ are the inertias along the $\psi$ and $\phi$ axes and $I_{m}$ and $I_{r}$ the inertias of the corresponding motors. A varying force in the horizontal (vertical) axis not only pushes the body in the horizontal (vertical) direction but also rotates the main (rear) motor. This effect is represented by the differential terms $I_{r} \ddot{\rho}_{r}$ and $I_{m} \ddot{\rho}_{m} \cdot u_{m}$ and $u_{r}$ are the two input currents and $K_{m}, K_{r}$ their corresponding electrical torque constants.

\subsection{Standard Decoupling}

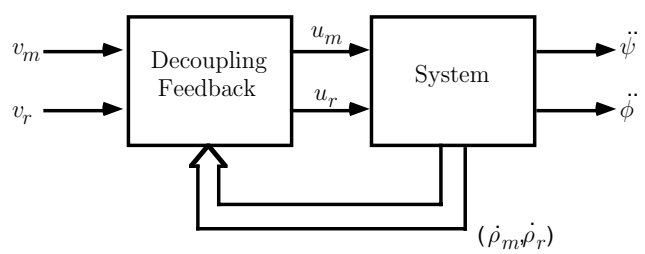

Figure 2. Decoupling Feedback

As shown in Figure 2, decoupling consists of choosing two new inputs $v_{m}$ and $v_{r}$ and feedback laws for $u_{m}$ and $u_{r}$ such that $\ddot{\psi}=v_{m}$ and $\ddot{\phi}=v_{r}$. For the system (1)-(4), the standard decoupling feedback is given by :

$$
\begin{aligned}
u_{r} & =\frac{C_{m}}{K_{r}} \dot{\rho}_{m}-\frac{I_{\psi}}{K_{r}} v_{m} \\
u_{m} & =\frac{C_{r}}{K_{m}} \dot{\rho}_{r}-\frac{I_{\phi}}{K_{m}} v_{r}
\end{aligned}
$$

With this control law, the internal dynamics of the two propeller speeds can be obtained by substituting (5)-(6) in $(3)-(4)$.

$$
\begin{gathered}
{\left[\begin{array}{c}
\ddot{\rho}_{m} \\
\ddot{\rho}_{r}
\end{array}\right]=\left[\begin{array}{cc}
0 & \frac{C_{r}}{I_{m}} \\
\frac{C_{m}}{I_{r}} & 0
\end{array}\right]\left[\begin{array}{c}
\dot{\rho}_{m} \\
\dot{\rho}_{r}
\end{array}\right]-\left[\begin{array}{cc}
\frac{I_{\phi}}{I_{m}} & 0 \\
0 & \frac{I_{\psi}}{I_{r}}
\end{array}\right]\left[\begin{array}{c}
v_{r} \\
v_{m}
\end{array}\right]} \\
\lambda\left(\left[\begin{array}{cc}
0 & \frac{C_{r}}{I_{m}} \\
\frac{C_{m}}{I_{r}} & 0
\end{array}\right]\right)= \pm \sqrt{\frac{C_{m} C_{r}}{I_{m} I_{r}}}
\end{gathered}
$$

Since one of the eigenvalues of the homogeneous part is in the right half plane, the internal dynamics is unstable. Looking from another point of view, (5)-(6) can be interpreted as controlling the $\psi$ axis with the rear motor and the $\phi$ axis with the main motor. Such a scheme makes little physical sense, and the fact that it is impractical is reflected in one of the eigenvalues being in the right half plane. 


\section{A DECOUPLING SCHEME IN DISCRETE TIME}

Let the controller be implemented in discrete time, with sampling period $T$. We will use the notation $x(k)$ for $x(t=k T)$. Also we will use $x\left(k_{+}\right)$to represent $x(t=$ $k T+\epsilon), \epsilon \downarrow 0$ and $x\left(k_{-}\right)$for $x(t=k T-\epsilon)$. For continuous states, $x\left(k_{-}\right)=x(k)=x\left(k_{+}\right)$. Decoupling in discrete time means that we should be able to choose inputs, constant during the interval, $k T \leq t<k T+T$, such that $\ddot{\psi}(k+d)=v_{m}(k)$ and $\ddot{\phi}(k+d)=v_{r}(k)$, where $k=$ $0,1,2, \ldots$ and $d:$ a delay. Note that we allow additional delays which we will exploit to obtain internally-stable decoupling. We will show that if $d=0$, stable decoupling is not possible, while a decoupling scheme can be worked out for $d=1$. Also, note that this definition imposes no constraints on the inter-sample behavior.

Since the motor equations are only a set of integrators, and since the inputs remain constant during the sampling interval, i.e., $u_{m}(k)=u_{m}\left(k_{+}\right)=u_{m}\left((k+1)_{-}\right)$ and $u_{r}(k)=u_{r}\left(k_{+}\right)=u_{r}\left((k+1)_{-}\right)$, equations (1)-(4) can be written in discrete time as follows :

$$
\begin{aligned}
I_{\psi} \ddot{\psi}(k) & =C_{m} \dot{\rho}_{m}(k)-K_{r} u_{r}(k) \\
I_{\phi} \ddot{\phi}(k) & =C_{r} \dot{\rho}_{r}(k)-K_{m} u_{m}(k) \\
\dot{\rho}_{m}(k+1) & =\dot{\rho}_{m}(k)+\frac{K_{m} T}{I_{m}} u_{m}(k) \\
\dot{\rho}_{r}(k+1) & =\dot{\rho}_{r}(k)+\frac{K_{r} T}{I_{r}} u_{r}(k)
\end{aligned}
$$

Equations (9) and (9) lead to the decoupling law :

$$
\begin{aligned}
u_{r}(k) & =\frac{C_{m}}{K_{r}} \dot{\rho}_{m}(k)-\frac{I_{\psi}}{K_{r}} v_{m}(k) \\
u_{m}(k) & =\frac{C_{r}}{K_{m}} \dot{\rho}_{r}(k)-\frac{I_{\phi}}{K_{m}} v_{r}(k)
\end{aligned}
$$

with $\ddot{\psi}(k)=v_{m}(k)$ and $\ddot{\phi}(k)=v_{r}(k)$. However, comparing (13)-(14) with (5)-(6), one can observe that this will also lead to unstable internal dynamics.

The decoupling problem is as follows : Equations (9)(10) have a direct term from one of the inputs and a term proportional to the integral of the other input. While it is possible to control $\ddot{\psi}$ and $\ddot{\phi}$ immediately using the direct terms, such a scheme leads to unstable internal dynamics. The core idea of the paper is to give relative importance to the integral terms over the direct terms. For this, we choose the control inputs such that $\ddot{\psi}$ and $\ddot{\phi}$ are equal to their desired values at the end rather than at the beginning of a sampling interval. This is what a designer would be forced to do in the absence of direct terms. Though internally-stable decoupling is possible, the presence of direct terms leads to a situation where this scheme works only for sampling intervals larger than a certain value.

Using the definition of the input to a sampled system and (9)-(12), $\ddot{\psi}$ and $\ddot{\phi}$ at time $(k+1)_{-}$are given by:

$$
\begin{aligned}
{\left[\begin{array}{c}
I_{\psi} \ddot{\psi}\left((k+1)_{-}\right) \\
I_{\phi} \ddot{\phi}\left((k+1)_{-}\right)
\end{array}\right] } & =\left[\begin{array}{c}
C_{m} \dot{\rho}_{m}(k) \\
C_{r} \dot{\rho}_{r}(k)
\end{array}\right]+\mathcal{B}\left[\begin{array}{c}
u_{m}(k) \\
u_{r}(k)
\end{array}\right] \\
\text { where } \mathcal{B} & =\left[\begin{array}{cc}
\frac{C_{m} K_{m} T}{I_{m}} & -K_{r} \\
-K_{m} & \frac{C_{r} K_{r} T}{I_{r}}
\end{array}\right]
\end{aligned}
$$

The decoupling law then reads :

$$
\left[\begin{array}{c}
u_{m}(k) \\
u_{r}(k)
\end{array}\right]=\mathcal{B}^{-1}\left[\begin{array}{c}
I_{\psi} v_{m}(k)-C_{m} \dot{\rho}_{m}(k) \\
I_{\phi} v_{r}(k)-C_{r} \dot{\rho}_{r}(k)
\end{array}\right]
$$

With such a controller, $\ddot{\psi}\left((k+1)_{-}\right)=v_{m}(k)$ and $\ddot{\phi}((k+$ $\left.1)_{-}\right)=v_{r}(k)$ for all $k$. Note that a unit delay is introduced in the process of decoupling. To study the internal stability of this input-output decoupled system, the control law (17) can be plugged back in (11)-(12) to give,

$$
\begin{aligned}
{\left[\begin{array}{c}
\dot{\rho}_{m}(k+1) \\
\dot{\rho}_{r}(k+1)
\end{array}\right]=} & {\left[\begin{array}{c}
\dot{\rho}_{m}(k) \\
\dot{\rho}_{r}(k)
\end{array}\right]+\left[\begin{array}{cc}
\frac{K_{m} T}{I_{m}} & 0 \\
0 & \frac{K_{r} T}{I_{r}}
\end{array}\right] } \\
& \mathcal{B}^{-1}\left[\begin{array}{c}
I_{\psi} v_{m}(k)-C_{m} \dot{\rho}_{m}(k) \\
I_{\phi} v_{r}(k)-C_{r} \dot{\rho}_{r}(k)
\end{array}\right]
\end{aligned}
$$

With $\mathcal{I}_{2}$ being the two-dimensional identity matrix, the homogeneous part of the internal dynamics and its eigenvalues can be written as :

$$
\begin{aligned}
{\left[\begin{array}{c}
\dot{\rho}_{m}(k+1) \\
\dot{\rho}_{r}(k+1)
\end{array}\right] } & =\mathcal{A}\left[\begin{array}{c}
\dot{\rho}_{m}(k) \\
\dot{\rho}_{r}(k)
\end{array}\right] \\
\text { where } \mathcal{A} & =\left(\mathcal{I}_{2}-\frac{T}{T^{2}-\tau^{2}}\left[\begin{array}{cc}
T & \frac{I_{r}}{C_{r}} \\
\frac{I_{m}}{C_{m}} & T
\end{array}\right]\right) \\
\lambda(\mathcal{A}) & =1-\frac{T}{T \pm \tau}=\frac{ \pm \tau}{T \pm \tau} \\
\text { and } \tau & =\sqrt{\frac{I_{m} I_{r}}{C_{m} C_{r}}}
\end{aligned}
$$

\subsection{Lower Bound on the Sampling Interval}

Theorem 1 : Internally-stable decoupling of the differentially cross-coupled system (9)-(12) is possible with the decoupling feedback given by (17), iff $T>2 \tau$, where $\tau$ is as given in (22).

Proof : From (21), it can be seen that one of the eigenvalues of $\mathcal{A}, \frac{\tau}{T+\tau}$, is always within the unit circle. If $T>2 \tau, T-\tau>\tau$ and hence the second eigenvalue $\frac{-\tau}{T-\tau}$ is also within the unit circle. Since both the eigenvalues of the internal dynamics are within the unit circle, the overall system is decoupled and stable. 
On the contrary, if $T \leq 2 \tau$, it can be seen from (21) that the second eigenvalue $\frac{-\tau}{T-\tau}$ is outside the open unit circle. This causes the internal dynamics to be either marginally stable or unstable, in which case, perfect internally-stable decoupling is not possible.

Remark 1: The aerodynamic term is proportional to the propeller speed, while the differential term is proportional to the acceleration given to the propeller. Clearly, when the inputs change, the acceleration changes immediately, while the speed does not do so. Hence, at the beginning of the sampling interval, the differential term always dominates. However, since the integral builds up with time, the forces proportional to the speeds become dominant after some time. Here, the sampling interval indirectly specifies the build-up time. Hence, the lower bound set by Theorem 1 can be interpreted as the minimum time required for the aerodynamic force to dominate the differential term.

Remark 2: It is interesting to note that the bound obtained by Theorem 1 (22), and the time constant of the unstable mode when standard input-output decoupling is performed (8) are numerically equal.

There are two important implications of Theorem 1. First, the fact that the system can be decoupled with stable internal dynamics. It becomes possible to implement noninteracting control if we are interested only in the values of the outputs at the sampling instants. This is sufficient to design feedback controllers with guaranteed stability, though the inter-sample evolution remains coupled. This will be the topic of discussion in the remaining part of this section. Secondly, Theorem 1 indicates that the sampling time should not be smaller than a certain limit. However, the bandwidth of the system together with Shannon's sampling theorem, imposes an upper limit, $T_{\max }$, on the sampling time. So the acceptable range of values for the sampling interval is restricted to $2 \tau<T<T_{\max }$. This interval might be too small for any practical implementation or the bounds may even be infeasible $\left(2 \tau>T_{\max }\right)$. In such a case, use of more sophisticated multi-time scale techniques has to be investigated.

\subsection{Controller Design}

For the remaining part of this paper, let us assume that the bounds are feasible, i.e., $\left(2 \tau<T_{\max }\right)$. So, by choosing a sampling interval in the feasible region, it is possible to use the feedback law (17) to decouple the system and reduce it to a pair of simple delays, $\ddot{\psi}\left((k+1)_{-}\right)=$ $v_{m}(k)$ and $\ddot{\phi}\left((k+1)_{-}\right)=v_{r}(k)$. Hence, one is left with choosing external stabilizing controllers for these simple delay systems as shown in Figure 3.

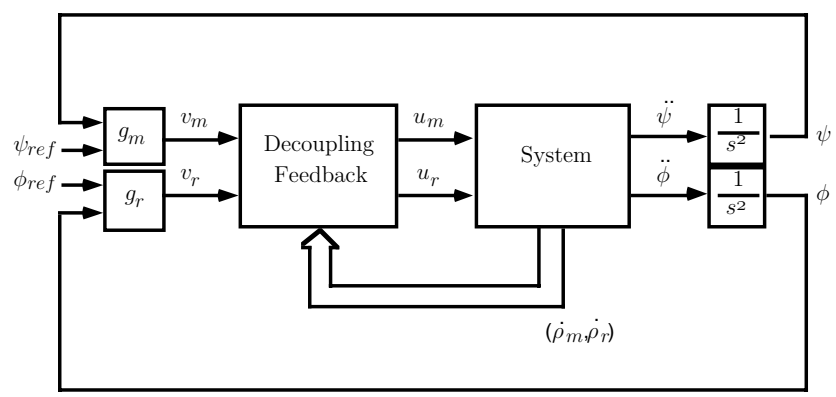

Figure 3. Controller Design with Decoupling

Theorem 2 : Let (i) the sampling interval be in the range $2 \tau<T<T_{\max }$, (ii) the controllers,

$$
v_{m}(k)=g_{m}\left(\psi_{r e f}, \psi, \dot{\psi}\right), v_{r}(k)=g_{r}\left(\phi_{r e f}, \phi, \dot{\phi}\right)
$$

with $\psi_{\text {ref }}$ and $\phi_{\text {ref }}$ being the corresponding reference inputs, stabilize the plants $\ddot{\psi}\left((k+1)_{-}\right)=v_{m}(k)$ and $\ddot{\phi}\left((k+1)_{-}\right)=v_{r}(k)$ respectively. Then, the decoupling feedback that stabilizes the plant (1)-(4) is given by :

$$
\left[\begin{array}{c}
u_{m}(k) \\
u_{r}(k)
\end{array}\right]=\mathcal{B}^{-1}\left[\begin{array}{c}
I_{\psi} g_{m}\left(\psi_{r e f}, \psi, \dot{\psi}\right)-C_{m} \dot{\rho}_{m}(k) \\
I_{\phi} g_{r}\left(\phi_{r e f}, \phi, \dot{\phi}\right)-C_{r} \dot{\rho}_{r}(k)
\end{array}\right]
$$

Proof : The feedback (24) is (23) substituted in (17). It was shown in Theorem 1 that, with a control law of the type $(17)$, it is possible to enforce any $\ddot{\psi}\left((k+1)_{-}\right)=$ $v_{m}(k)$ and $\ddot{\phi}\left((k+1)_{-}\right)=v_{r}(k)$ maintaining internal stability. Since by hypothesis (i), the controllers $g_{m}$ and $g_{r}$ stabilize the delay systems, the proof is complete, if we show that the inter-sample behavior does not affect stability. Inter-sample evolution is important since in the system resulting from the decoupling step, $\ddot{\psi}$ and $\ddot{\phi}$ do not remain constant between samples.

Note that the inter-sample oscillations, if any, will have a frequency above $1 /(2 T) . T_{\max }$ is twice the inverse of the bandwidth of the system (bandwidth $=1 /\left(2 T_{\max }\right)$ ). Since the inter-sample oscillations have frequencies larger than $1 /(2 T)>1 /\left(2 T_{\max }\right)$, by definition of the bandwidth, their loop gain will be strictly less than 1 . Hence from the small gain theorem (Vidyasagar 1993), the inter-sample behavior will not affect the stability of the overall system.

\section{THE COMPLETE MODEL}

The complete model of the experimental setup described in Section 2, is derived using the Lagrange method (Mullhaupt 1994) and is given below : 


$$
\begin{aligned}
& I_{\psi} \ddot{\psi}+I_{r} \ddot{\rho}_{r}=C_{m} \dot{\rho}_{m} \\
& \quad+I_{m} \cos (\psi) \dot{\phi} \dot{\rho}_{m}+\frac{1}{2} I_{c}(\dot{\phi})^{2} \sin (2 \psi) \\
& \quad-C_{r 1} \dot{\rho}_{r}-F_{\psi} \dot{\psi}-G_{s} \sin (\psi)-G_{c} \cos (\psi) \\
& \left(I_{\phi}+I_{c} \sin ^{2}(\psi)\right) \ddot{\phi}+I_{m} \sin (\psi) \ddot{\rho}_{m}=C_{r} \dot{\rho}_{r} \\
& \quad-I_{m} \cos (\psi) \dot{\psi} \dot{\rho}_{m}-I_{c} \sin (2 \psi) \dot{\psi} \dot{\phi} \\
& \quad-C_{m 1} \sin (\psi) \dot{\rho}_{r}-F_{\phi} \dot{\phi} \\
& I_{m} \ddot{\rho}_{m}=K_{m} u_{m}-\left(F_{m}+C_{m 1}\right) \dot{\rho}_{m} \\
& \quad-I_{m}(\cos (\psi) \dot{\psi} \dot{\phi}+\ddot{\phi} \sin (\psi)) \\
& I_{r} \ddot{\rho}_{r}=K_{r} u_{r}-\left(F_{r}+C_{r 1}\right) \dot{\rho}_{r}-I_{r} \ddot{\psi}
\end{aligned}
$$

- $C_{m 1}, C_{r 1}$ : aerodynamic cross-coupling constants.

- $I_{c}$ : Inertia corresponding to the Coriolis term.

- $G_{s}, G_{c}$ : gravity effect constants.

- $F_{\psi}, F_{\phi}, F_{m}, F_{r}$ : viscous friction constants.

The complete model contains aerodynamic cross-coupling terms, coriolis and centrifugal terms, viscous friction, gravity effects and a projection necessitated by geometrical constraints. Most of the terms do not affect the theorems developed in Section 3. The effect of various terms is stated in the corollaries that follow, the proofs being omitted for brevity.

Corollary 1: Given the system

$$
\begin{aligned}
I_{\psi} \ddot{\psi}+I_{r} \ddot{\rho}_{r} & =C_{m} \dot{\rho}_{m}+f_{m}(\psi, \dot{\psi}, \phi, \dot{\phi}) \\
I_{\phi} \ddot{\phi}+I_{m} \ddot{\rho}_{m} & =C_{r} \dot{\rho}_{r}+f_{r}(\psi, \dot{\psi}, \phi, \dot{\phi}) \\
I_{m} \ddot{\rho}_{m} & =K_{m} u_{m} \\
I_{r} \ddot{\rho}_{r} & =K_{r} u_{r}
\end{aligned}
$$

with $f_{m}$ and $f_{r}$ being arbitrary nonlinear functions, the system can be decoupled with internal stability iff $T>$ $2 \tau$, with $\tau$ being given by (22). The decoupling feedback

$$
\left[\begin{array}{c}
u_{m}(k) \\
u_{r}(k)
\end{array}\right]=\mathcal{B}^{-1}\left[\begin{array}{c}
I_{\psi} v_{m}(k)-C_{m} \dot{\rho}_{m}(k)-f_{m}(k) \\
I_{\phi} v_{r}(k)-C_{r} \dot{\rho}_{r}(k)-f_{r}(k)
\end{array}\right]
$$

leads to $\ddot{\psi}\left((k+1)_{-}\right)=v_{m}(k)$ and $\ddot{\phi}\left((k+1)_{-}\right)=v_{r}(k)$.

The result essentially states that the gravity, viscous friction and coriolis terms involving only $(\dot{\psi}, \dot{\phi})$ do not change the lower bound. Also, the nonlinearities of this type can be easily compensated for, leading to a simple time-invariant system.

Corollary 2: The system

$$
\begin{aligned}
I_{\psi} \ddot{\phi}+I_{r} \ddot{\rho}_{r} & =C_{m} \dot{\rho}_{m}-C_{r 1} \dot{\rho}_{r}+f_{m}(\psi, \dot{\psi}, \phi, \dot{\phi}) \\
I_{\phi} \ddot{\phi}+I_{m} \ddot{\rho}_{m} & =C_{r} \dot{\rho}_{r}-C_{m 1} \dot{\rho}_{m}+f_{r}(\psi, \dot{\psi}, \phi, \dot{\phi}) \\
I_{m} \ddot{\rho}_{m} & =K_{m} u_{m}-\left(F_{m}+C_{m 1}\right) \dot{\rho}_{m} \\
I_{r} \ddot{\rho}_{r} & =K_{r} u_{r}-\left(F_{r}+C_{r 1}\right) \dot{\rho}_{r}
\end{aligned}
$$

can be decoupled with internal stability iff $T>2 \tau_{1}$, with $\tau_{1}$ being a constant expressible as function of model parameters. A decoupling feedback similar to (33) leads to $\ddot{\psi}\left((k+1)_{-}\right)=v_{m}(k)$ and $\ddot{\phi}\left((k+1)_{-}\right)=v_{r}(k)$.

With the introduction of aerodynamic cross-coupling terms and the viscous friction terms in the motor equations, the expression for the lower bound becomes complicated but the bound is not a function of the states. Though the feedback law is more involved, the nonlinearities can be compensated for.

However, when terms of the form $\dot{\phi} \dot{\rho}_{m}$ as in (25)-(26) are added, the homogeneous system analogous to (19) becomes time-varying. Hence, an obvious lower bound on the sampling interval can no longer be obtained. Yet, the decoupling feedback can be computed and leads to the well-analyzed system, $\ddot{\psi}\left((k+1)_{-}\right)=v_{m}(k)$ and $\ddot{\phi}\left((k+1)_{-}\right)=v_{r}(k)$. So, the results on controller design still hold. Such a controller is designed for the complete model and the fact that the system can be decoupled with a sufficiently large sampling period is demonstrated in simulation. The relevance of the lower bound on $T$ is also illustrated.

\section{SIMULATIONS}

Simulation of the complete model controlled by the decoupling feedback developed in the lines of (33) was performed. The parameters of the model were obtained from the experimental setup by direct measurement of the masses and distances and other simple experiments. The bandwidth of the linearized system was in the order of a few hertz and Shannon's limit was around $T_{\max } \approx$ $200 \mathrm{~ms}$. As was noted, since the internal dynamics is time-varying, analytical expressions on the lower bound are not available. From simulations it was found that the lower bound with internal stability was around $2 \tau \approx$ $2 \mathrm{~ms}$. The first simulation, shown in Figure 4, was performed for $T=10 \mathrm{~ms}$. A simple PD controller, with the proportional gain $K_{p}=4$ and the derivative gain $K_{d}=4$, was used to generate $v_{m}$ and $v_{r}$. Nominal zeromean gaussian process noise was also added to make the simulations behave closer to reality.

The set points and actual values of $\psi$ and $\phi$ are shown in Figure 4. It can be seen that the dynamics of the two axes are essentially decoupled. Changes in the set point of one axis does not affect the evolution of the other axis. Also the responses are monotonic without oscillations. Such responses cannot be achieved when simple PD controllers are used for either axis. Note that the speeds do not blow up, an indication of internal stability. 
If the sampling interval is reduced to $1 \mathrm{~ms}$, the internal dynamics go unstable. As can be seen in Figure 5, the speeds go well beyond their limits. Though the internal dynamics goes unstable, the variables $\psi$ and $\phi$ would have continued their evolution as in Figure 4 in the absence of process noise. However, the added noise forces the entire system to go unstable. This clearly substantiates the necessity of a lower bound on the sampling interval, which emphasizes the theoretical results presented in this paper.
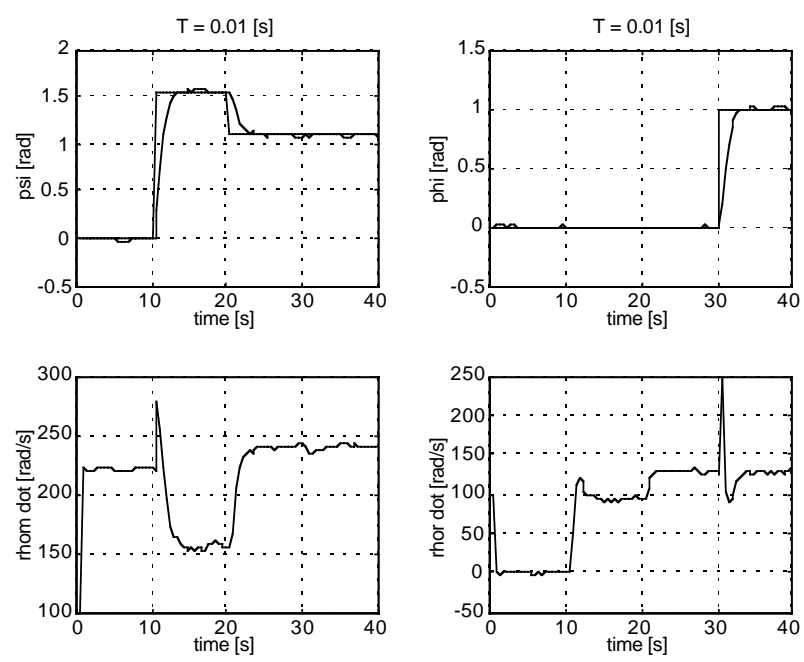

Figure 4. Simulation Results with $T=10 \mathrm{~ms}$
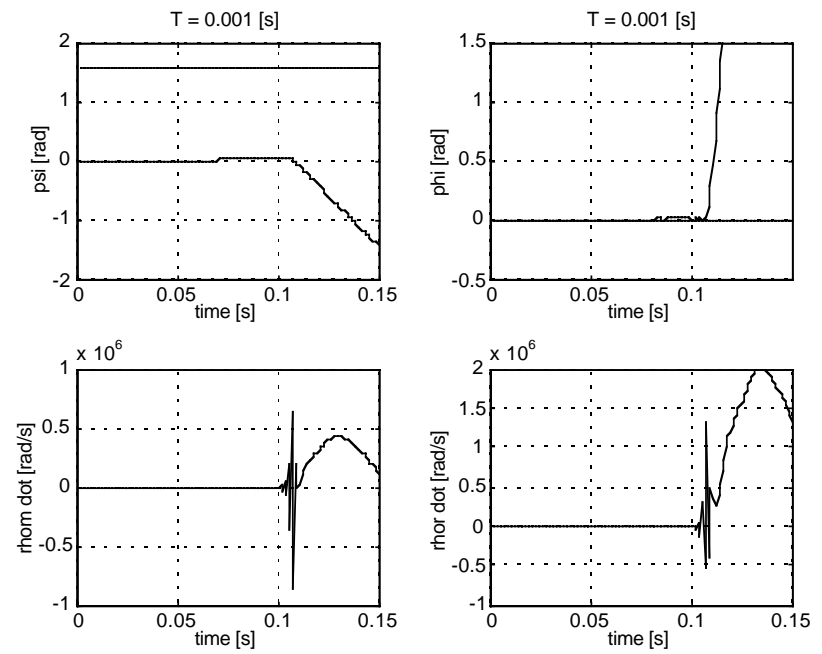

Figure 5. Simulation Results with $T=1 \mathrm{~ms}$

\section{CONCLUSIONS}

The problem of internally-stable decoupling in differentially cross-coupled systems is addressed. A model helicopter in which the speed is varied to control the aero- dynamic force was considered as a motivation and case study. A discrete-time decoupling law was proposed which leads to internally-stable decoupling. It was shown that the sampling interval should be bounded from below for this scheme to work. Design of a stabilizing controller with such a decoupling feedback was also addressed. Finally, simulation results which substantiate the theoretical developments were presented.

Two open problems that immediately arise from this work are as follows : (i) With the addition of coriolis terms, the homogeneous system becomes time-varying. How can the stability of this scheme be proved analytically ? (ii) If Shannon's limit and the lower bound indicated in this work lead to an infeasible region for the sampling interval, how can a controller be designed ? These are a few among the many problems that should be addressed towards the goal of understanding differentially cross-coupled systems.

\section{REFERENCES}

Byrnes, C.I., A. Isidori and J.C. Willems (1991). Passivity, feedback equivalence, and the global stabilization of minimum phase systems. IEEE Transactions Automatic Control 36(11), 1228-1240.

Fliess, M., J. Levine, P. Martin and P. Rouchon (1995). Flatness and defect of non-linear systems: introductory theory and examples. International Journal of Control 61(6), 1327-1361.

Franklin, G.F., J.D. Powell and M.L. Workman (1990). Digital Control of Dynamic System. second ed.. Addsion-Wesley.

Isidori, A., A.J. Krener, C. Gori-Giorgi and S. Monaco (1981). Nonlinear decoupling via feedback: A differential geometric approach. IEEE Transactions Automatic Control 26(2), 331-345.

Kokotovic, P. (1992). The joy of feedback. Control Systems Magazine 12(3), 7-17.

Meyer, G., R. Su and L.R. Hunt (1984). Application of nonlinear transformations to automatic flight control. Automatica 20(1), 102-107.

Mullhaupt, P. (1994). Modélisation et identification d'un modèle réduit d'hélicoptère. Master's thesis. Université de Paris-Sud (Orsay).

Nijmeier, H. and A.J. van der Schaft (1990). Nonlinear Dynamical Control Systems. Springer. New york.

Ortega, R. and M.W. Spong (1989). Adaptive motion control of rigid robots: a tutorial. Automatica 26(6), 877-888.

Prouty, R.W. (1986). Helicopter Performance, Stability and Control. PWS Engineering. Boston.

Vidyasagar, M. (1993). Nonlinear Systems Analysis. second ed.. Prentice-Hall International. 